Pacific Journal of Mathematics

FREE TOPOLOGICAL SEMIGROUPS AND EMBEDDING
TOPOLOGICAL SEMIGROUPS IN TOPOLOGICAL GROUPS 


\title{
FREE TOPOLOGICAL SEMIGROUPS AND EMBEDDING TOPOLOGICAL SEMIGROUPS IN TOPOLOGICAL GROUPS
}

\author{
Francis T. Christoph, JR.
}

The purpose of this paper is to study the problem of embedding a topological semigroup in a topological group. A construction is given for a free topological semigroup generated by a topological space. This construction is used to define a concept called Property $E$ and it is shown that a $T_{1}$ completely regular topological semigroup $S$ can be embedded in a topological group if and only if $S$ can be embedded in a group and $S$ has Property $E$. This generalizes a result of Rothman who considered the problem of embedding a commutative, cancellative topological semigroup in its group of quotients. Rothman's results on embedding certain subsemigroups of compact semigroups in topological groups are also generalized.

Free topological groups have been constructed and studied by a number of people (e.g., see [4]). In this paper we give a construction for a free topological semigroup (1.1). This construction resembles the one in [4] for topological groups. In $\S 2$ free topological semigroups and free topological groups are used to investigate the problem of embedding a topological semigroup in a topological group. Rothman [7] defined a concept called Property $F$ and proved that Property $F$ is a necessary and sufficient condition for embedding a $T_{2}$ commutative, cancellative topological semigroup in its group of quotients as an open subset. Rothman's result is generalized by defining Property $E$ and proving that a $T_{1}$ completely regular topological semigroup $S$ can be embedded in a topological group by a topological isomorphism if and only if $S$ can be embedded (algebraically) in a group and $S$ has Property $E$. Examples are given to illustrate the differences between Property $F$ and Property $E$. Rothman [7] also defined a concept called Property $J$ and showed that certain subsemigroups having Property $F$ or Property $J$ of compact semigroups could be embedded in a topological group. His results are generalized in $\S 3$ by introducing group liftable semigroups and showing that certain group liftable subsemigroups of compact Hausdorff semigroups are embeddable in a topological group.

A topological semigroup is a topological space with a continuous, associative multiplication. A continuous function will be called a map. The cardinality of a set $Y$ will be denoted by $|Y|$. Let $C$ be the cardinality of the continuum. 


\section{Free topological semigroups.}

THEOREM 1.1. If $X$ is a topological space, then there exists a topological semigroup $F(X)$ such that $X$ is embedded in $F(X)$ by a homeomorphism and such that if $f: X \rightarrow S$ is a map of $X$ into a topological semigroup $S$, then $f$ can be extended to a continuous homomorphism $f^{\prime}$ of $F(X)$ into $S$.

Proof. Let $E$ be a collection of topological semigroups $S$ such that $|S| \leqq \max (|X|, C)$, distinct members of $E$ are not topologically isomorphic, and each topological semigroup $S^{\prime}$ with $\left|S^{\prime}\right| \leqq \max (|X|, C)$ is topologically isomorphic with some $S \in E$. Let $\left\{\left(S_{\alpha}, f_{\alpha}\right)\right\}_{\alpha \in K}$ consist of all pairs $\left(S_{\alpha}, f_{\alpha}\right)$ such that $S_{\alpha} \in E$ and $f_{\alpha}$ is a map of $X$ into $S_{\alpha}$. If $S^{\prime}$ is a topological semigroup and $g: X \rightarrow S^{\prime}$ is a map with $\left|S^{\prime}\right| \leqq$ $\max (|X|, C)$, then there is an $\alpha_{0} \in K$ such that $S^{\prime}$ is topologically isomorphic to $S_{\alpha_{0}}$ by a topological isomorphism $h: S_{\alpha_{0}} \rightarrow S^{\prime}$ such that $g=h f_{\alpha_{0}}$. Identify $\left(S^{\prime}, g\right)$ with $\left(S_{\alpha_{0}}, f_{\alpha_{0}}\right)$. Define $\mathscr{O}: X \rightarrow \prod_{\alpha \in K} S_{\alpha}$ by $(\mathscr{O}(x))(\alpha)=f_{\alpha}(x)$ for $x \in X$. We now show that $\mathscr{O}$ is a homeomorphism. The continuity of $\mathscr{O}$ follows from the fact that $p_{\alpha} \mathscr{O}=f_{\alpha}$ where $p_{\alpha}$ : $\prod_{\alpha \in K} S_{\alpha} \rightarrow S_{\alpha}$ is the projection into the $\alpha$-th coordinate. The identity function $i: X \rightarrow X$ is continuous and $X$ is a topological semigroup with right zero multiplication: $x y=y$ for $x, y \in X$. If $a \in X$ and $B$ is a closed subset of $X$ with $a \notin B$, then $i(a)$ is not in the closure of $B$. Hence $\left\{f_{\alpha}\right\}_{\alpha \in K}$ distinguishes points and closed sets. Thus $\mathcal{O}$ is one-toone and open. Identify $X$ with $\mathscr{O}(X)$. Let $F(X)$ be the subsemigroup of $\prod_{\alpha \in K} S_{\alpha}$ generated by $\mathscr{O}(X)$. Now let $f: X \rightarrow S$ be a map of $X$ into a topological semigroup $S$. The semigroup $S g[f(X)]$ generated by $f(X)$ has cardinality less than or equal to $\max (|X|, C)$. Thus $(S g[f(X)], f)$ is identified with $\left(S_{\alpha_{0}}, f_{\alpha_{0}}\right) \in\left\{\left(S_{\alpha}, f_{\alpha}\right)\right\}_{\alpha \in K}$ for some $\alpha_{0} \in K$. Therefore $f$ is projection into the $\alpha_{0}$-th coordinate and can be extended to a continuous homomorphism of $F(X)$ into $S g[f(X)]$.

Definition 1.2. The pair $(F(X), \mathscr{O})$ is called the free topological semigroup generated by $X$. (We will sometimes not mention $\mathscr{O}$ explicitly and will call $F(X)$ the free topological semigroup generated by $X$.)

Proposition 1.3. Algebraically $F(X)$ is the free semigroup generated by $\mathscr{O}(X)$.

Proof. Let $f: X \rightarrow S$ be a function into an algebraic semigroup $S$. Give $S$ the indiscrete topology to obtain a topological semigroup such that $f$ is continuous. By 1.1 there exists a continuous homomorphism $f^{\prime}: F(X) \rightarrow S$ such that $f=f^{\prime} O$. By Theorem 9.4 of [1] 
we have that $F(X)$ is the free semigroup generated by $\mathscr{O}(X)$.

REMARK 1.4. If we consider only $T_{2}$ semigroups in the construction of $F(X)$, then $F(X)$ is $T_{2}$ and the map extension property holds for $T_{2}$ semigroups.

Proposition 1.5. Every topological semigroup $S$ is an algebraic quotient semigroup of a free topological semigroup such that the natural projection homomorphism is continuous.

Proof. Let $i: S \rightarrow S$ be the identity function. By $1.1 i$ can be extended to a continuous homomorphism $i^{\prime}$ of $F(S)$ onto $S$. Let $R$ be the congruence relation associated with $i^{\prime}$. We have that $F(S) / R$ is algebraically isomorphic to $S$ and the natural projection $p: F(S) \rightarrow$ $F(S) / R$ is continuous.

If the map $i^{\prime}$ in the proof of 1.5 is open, then $F(S) / R$ is a topological semigroup and is topologically isomorphic to $S$.

2. Embedding topological semigroups in topological groups. It is a well-known algebraic result that a commutative, cancellative semigroup can be embedded in a group (called the group of quotients or the group generated by the semigroup). In [7] Rothman considers the problem of embedding (by a topological isomorphism) a commutative, cancellative $T_{2}$ topological semigroup in its group of quotients (furnished with the quotient topology) as an open subset. Rothman defines the following concept and proves that it is a necessary and sufficient condition for the solution of his embedding problem.

Definition 2.1. A topological semigroup $S$ has Property $F$ if and only if $x, y \in S$ and $V$ an open subset of $S$ containing $x$ imply there exists an open set $W$ with $y \in W$ such that $x y \in \bigcap_{y^{\prime} \in W} V y^{\prime}$ and $y x \in \bigcap_{y^{\prime} \in W} y^{\prime} V$.

In the following we generalize Rothman's problem and solution by finding necessary and sufficient conditions for a $T_{1}$ completely regular topological semigroup to be embedded in a topological group by a topological isomorphism. Free topological semigroups and free topological groups are important tools for investigating this problem.

Let $Z_{2}$ be the two element semigroup $\{+1,-1\}$ with binary operation defined by $(+1)(-1)=(-1)(+1)=(+1)(+1)=(-1)(-1)=+1$. For a positive integer $i$ let $Z(i)=\{1,2, \cdots, i\}$.

Let $S$ be a semigroup and $A$ a directed set. If for each $a \in A$ we have a finite sequence $s_{a, i, 1}, s_{a, i, 2}, \cdots, s_{a, i, r(a, i)}$ of $r(a, i)$ many ele- 
ments in $S$ for $i=1,2, \cdots, n(a)$ where $n(\alpha)$ depends on $a \in A, r(a, i)$ depends on $a$ and on $i$, then let

$$
s(a, i, r)=\left\{s_{a, i, 1}, s_{a, i, 2}, \cdots, s_{a, i, r(a, i)}\right\}
$$

for $a \in A, i \in Z(n(a))$. The collection $\{s(a, i, r): a \in A, i \in Z(n(a))\}$ will be called a $f$-sequence in $S$. If $\{s(a, i, r): a \in A, i \in Z(n(a))\}$ is a $f$-sequence in $S$ and $\{d(a, i, r): a \in A, i \in Z(n(a))\}$ is a $f$-sequence in $Z_{2}$, then the pair

$$
(\{s(a, i, r): a \in A, i \in Z(n(a))\},\{d(a, i, r): a \in A, i \in Z(n(a))\})
$$

will be called a signed $f$-sequence and will be denoted by

$$
\left\{\left(s(a, i, r), d^{s}\right): a \in A, i \in Z(n(a))\right\} .
$$

If $\left\{\left(s(a, i, r), d^{s}\right): a \in A, i \in Z(n(a))\right\}$,

$$
\left.\left\{t(a, i, j), d^{t}\right): a \in A, i \in Z(n(a))\right\}, \cdots,\left\{u(a, i, p), d^{u}: a \in A, i \in Z(n(a))\right\}
$$

is a finite collection of signed $f$-sequences in $S$ with $i$ ranging over the same set $Z(n(\alpha))$ for each $a \in A$ in each $f$-sequence, then this collection of $f$-sequences will be denoted more briefly by

$$
\{s(\alpha, i, r), t(a, i, j), \cdots, u(\alpha, i, p): a \in A, i \in Z(n(\alpha))\} .
$$

For the remainder of $\S 2$ let $S$ be a $T_{1}$ completely regular topological semigroup and let $\left\{\left(S_{\alpha}, f_{\alpha}\right)\right\}_{\alpha \in K}$ be the collection given in the proof of 1.1 which corresponds to the free topological semigroup $F(S)$ generated by $S$. Let $e^{\prime}$ be the evaluation map corresponding to $K$, i.e., $e^{\prime}$ : $S \rightarrow \prod_{\alpha \in K} S_{\alpha}$ is defined by $\left(e^{\prime}(s)\right)(\alpha)=f_{\alpha}(s)$ for $s \in S$. In a similar manner we can also obtain a collection $\left\{\left(G_{i}, k_{\hat{o}}\right)\right\}_{\hat{o} \in D}, G_{o}$ a topological group for each $\delta \in D=D(S)$ by replacing "semigroup" by "group" in the construction of the proof of 1.1. In Theorem 8.8 of [3] this last collection is used to construct a free topological group $(G(S), e)$ generated by $S$ where $e$ is the evaluation corresponding to $D$. If $\left\{s(a, i, r), d^{s}\right)$ : $a \in A, i \in Z(n(a))\}$ is a signed $f$-sequence in $S$, then let

$$
e\left(s(a, i, r), d^{s}\right)=\left(e\left(s_{a, i, 1}\right)\right)^{d} a, i, 1\left(e\left(s_{a, i, 2}\right)\right)^{d} a, i, 2 \cdots\left(e\left(s_{a, i, r(a, i)}\right)\right)^{d}{ }_{a, i, r(a, i)} .
$$

Property $E$ is defined below in 2.2. This concept plays the role of Rothman's Property $F$ in our investigation. Property $E$ is best understood by examining it in the framework of the proof of 2.4 below. It is interesting to compare Property $E$ with Malcev's [5] necessary and sufficient conditions for embedding a semigroup in a group.

Definition 2.2. The topological semigroup $S$ has Property $E$ if and only if the existence of a directed set $A$, signed $f$-sequences 


$$
\begin{gathered}
\left\{\left(s(a, i, r), d^{s}\right),\left(t(a, i, j), d^{t}\right),\left(t^{\prime}\left(a, i, j^{\prime}\right), d^{t^{\prime}}\right),\left(u(a, i, m), d^{u}\right),\right. \\
\left.\left(u^{\prime}\left(a, i, m^{\prime}\right), d^{u^{\prime}}\right): a \in A, i \in Z(n(a)+1)\right\}
\end{gathered}
$$

$f$-sequences

$$
\{v(a, i, p): a \in A, i \in Z(n(a)+1)\},\left\{v^{\prime}\left(a, i, p^{\prime}\right): a \in A, i \in Z(n(a)+1)\right\},
$$

a net $\left\{s_{a}, a \in A\right\}$ in $S$, and $s \in S$ such that (1)-(4), (6)-(7) below hold for each $a \in A, i \in Z(n(a)+1)$ and such that (5) below holds for each $a \in A, i \in Z(n(a))$ imply that $k_{\delta}\left(s_{a}\right) \rightarrow k_{\delta}(s)$ for each $\delta \in D=D(S)$.

$$
\begin{gathered}
v_{a, i, 1} v_{a, i, 2} \cdots v_{a, i, p(a, i)}=v_{a, i, 1}^{\prime} v_{a, i, 2}^{\prime} \cdots v_{a, i, p^{\prime}(a, i)}^{\prime}, \\
e\left(v_{a, i, 1}\right) e\left(v_{a, i, 2}\right) \cdots e\left(v_{a, i, p(a, i)}\right)=e\left(u(a, i, m), d^{u}\right), \\
e\left(v_{a, i, 1}^{\prime}\right) e\left(v_{a, i, 2}^{\prime}\right) \cdots e\left(v_{a, i, p^{\prime}(a, i)}^{\prime}\right)=e\left(u^{\prime}\left(a, i, m^{\prime}\right), d^{u^{\prime}}\right), \\
e\left(s(a, i, r), d^{s}\right)=e\left(t(a, i, j), d^{t}\right) e\left(u(a, i, m), d^{u}\right) e\left(t^{\prime}\left(a, i, j^{\prime}\right), d^{t^{\prime}}\right), \\
e\left(s(a, i+1, r), d^{s}\right)=e\left(t(a, i, j), d^{t}\right) e\left(u^{\prime}\left(a, i, m^{\prime}\right), d^{u^{\prime}}\right) e\left(t^{\prime}\left(a, i, j^{\prime}\right), d^{t^{\prime}}\right), \\
e\left(s_{a}\right)=e\left(t(a, n(a)+1, j), d^{t}\right) e\left(u^{\prime}\left(a, n(a)+1, m^{\prime}\right), d^{u^{\prime}}\right) \\
\times e\left(t^{\prime}\left(a, n(a)+1, j^{\prime}\right), d^{t^{\prime}}\right), \\
k_{\delta}\left(s_{a, 1,1}\right)^{d a, 1,1} k_{\hat{\delta}}\left(s_{a, 1,2}\right)^{d a, 1,2} \cdots \\
k_{\delta}\left(s_{a, 1, r(a, 1)}\right)^{d a, i, r(a, 1)} \longrightarrow k_{\hat{o}}(s) \text { for each } \delta \in D .
\end{gathered}
$$

If $M$ is a semigroup and $R$ is a relation on $M$ (i.e., a subset of $M \times M)$, then the congruence $R^{\prime}$ on $M$ generated by $R$ [1] is the intersection of all congruences on $M$ containing $R$. In Theorem 1.8 of [1] there is an explicit description of $R^{\prime}$ in terms of $R$ as follows. Let $R_{1}=R \cup R^{-1} \cup \Delta$ where $R^{-1}$ is the relation on $M$ defined by $\left(m_{1}, m_{2}\right) \in R^{-1}$ for $m_{1}, m_{2} \in M$ if and only if $\left(m_{2}, m_{1}\right) \in R$ and $\Delta$ is the equality relation defined by $\left(m_{1}, m_{2}\right) \in \Delta$ if and only if $m_{1}=m_{2} \in M$. For $m_{1}, m_{2} \in M$ define the relation $R_{2}$ on $M$ by $\left(m_{1}, m_{2}\right) \in R_{2}$ if and only if there exist $c_{1}, c_{2}, c_{3}, c_{4} \in M$ such that $m_{1}=c_{3} c_{1} c_{4}, m_{2}=c_{3} c_{2} c_{4}$, and $\left(c_{1}, c_{2}\right) \in R_{1}$. Finally, define $R^{\prime}$ by $\left(m_{1}, m_{2}\right) \in R^{\prime}$ for $m_{1}, m_{2} \in M$ if and only if there exist $w_{1}, w_{2}, \cdots, w_{n} \in M$ such that $\left(m_{1}, w_{1}\right),\left(w_{1}, w_{2}\right), \cdots$, $\left(w_{n-1}, w_{n}\right),\left(w_{n}, m_{2}\right) \in R_{2}$. This explicit description of the congruence generated by a relation will be used in the proof of Theorem 2.4 below.

The following algebraic result may be found in [1, p. 294].

THEOREM 2.3. Let $M$ be a semigroup, $P$ a set of generators of $M$, and $i_{P}: P \rightarrow M$ the inclusion function. Let $(T, f)$ be a free semigroup on $P$ and $(G, k)$ a free group on $P$. Let $w: T \rightarrow G$ be the homomorphism such that $w f=k$ and $q: T \rightarrow M$ the homomorphism such 
that $q f=i_{P}$. Set $R=w^{-1} \circ q \circ q^{-1} \circ w$ and let $R^{\prime}$ be the congruence on $G$ generated by $R$. If $\lambda: M \rightarrow G / R^{\prime}$ is the homomorphism such that $\lambda i_{P}=p_{R^{\prime}}$ k where $p_{R^{\prime}}: G \rightarrow G / R^{\prime}$ is the projection, then $M$ can be embedded in a group if and only if $\lambda$ is an embedding.

We now have our main theorem.

Theorem 2.4. The topological semigroup $S$ can be embedded in a topological group by a topological isomorphism if and only if $S$ can be embedded (algebraically) in a group and $S$ has Property E.

Proof. Assume $S$ can be embedded (algebraically) in a group and $S$ has Property $E$. Let $\left(F(S), e^{\prime}\right)$ be the free topological semigroup generated by $S$ constructed in 1.1. Note that $S$ is embedded topologically in $F(S)$ by $e^{\prime}$. Let $(G(S), e)$ be the free topological group generated by $S$. We have that $S$ is embedded topologically in $G(S)$ by $e$ and that $G=G(S)$ is algebraically the free group generated by $S$. Let $i_{S}: S \rightarrow S$ be the identity function on $S$. By 1.1 there exist continuous homeomorphisms $q: F(S) \rightarrow S$ and $w: F(S) \rightarrow G$ such that $q e^{\prime}=i_{S}$ and $w e^{\prime}=e$. Let $R=w^{-1} \circ q \circ q^{-1} \circ w, R^{\prime}$ the congruence generated by $R$, and $p_{R^{\prime}}: G \rightarrow G / R^{\prime}$ the natural projection. We have that $F(S)$ is algebraically free by 1.3. It follows from discrete semigroup theory (see $\left[1\right.$, p. 294]) that $p_{R^{\prime}} e: S \rightarrow G / R^{\prime}$ is a one-to-one homomorphism into $G / R^{\prime}$, a topological group. Now $p_{R^{\prime}}, e$ is continuous since both $e$ and $p_{R^{\prime}}$, are continuous. To show that $p_{R^{\prime}} e: S \rightarrow p_{R^{\prime}} e(S)$ is open, let $x_{b} \rightarrow x$ be a convergent net in $p_{R^{\prime}} e(S)$. Note that $p_{R^{\prime}}$ is open and hence there exists a subnet $\left\{x_{a}, a \in A\right\}$ of $\left\{x_{b}\right\}$ and a net $\left\{g_{a}, a \in A\right\}$ converging to $g$ in $G$ such that $g=e\left(p_{R^{\prime}}, e\right)^{-1} x, p_{R^{\prime}}\left(g_{a}\right)=x_{a}$ for each $a \in A$, and $\left(g_{a}\right.$, $\left.e\left(p_{R^{\prime}} e\right)^{-1} x_{a}\right) \in R^{\prime}$ for each $a \in A$. In the definition of Property $E$ let $s=e^{-1}(g)$ and $s_{a}=\left(p_{R^{\prime}}, e\right)^{-1} x_{a}$ for each $a \in A$. Notice that $R=R \cup R^{-1} \cup \Delta$. Define a relation $R_{2}$ on $G$ by $\left(z_{1}, z_{2}\right) \in R_{2}$ for $z_{1}, z_{2} \in G$ if and only if there exist $z_{1}^{\prime}, z_{2}^{\prime}, z_{3}^{\prime}, z_{4}^{\prime} \in G$ such that $z_{1}=z_{3}^{\prime} z_{1}^{\prime} z_{4}^{\prime}, z_{2}=z_{3}^{\prime} z_{2}^{\prime} z_{4}^{\prime}$, and $\left(z_{1}^{\prime}, z_{2}^{\prime}\right) \in R$. Since $\left(g_{a}, e\left(s_{a}\right)\right) \in R^{\prime}$ for each $a \in A$, there exist $g_{a}=g_{a, 1}, g_{a, 2}, \cdots, g_{a, n(a)}$, $g_{a, n(a)+1}$ in $G$ for each $a \in A$ such that

$$
\left(g_{a, 1}, g_{a, 2}\right),\left(g_{a, 2}, g_{a, 3}\right), \cdots,\left(g_{a, n(a)}, g_{a, n(a)+1}\right),\left(g_{a, n(a)+1}, e\left(s_{a}\right)\right) \in R_{2}
$$

for each $a \in A$. There exists a signed $f$-sequence $\left\{\left(s(a, i, r), d^{s}\right): a \in A\right.$, $i \in Z(n(a)+1)\}$ in $S$ such that $g_{a, i}=e\left(s(a, i, r), d^{s}\right)$ for $a \in A, i \in Z(n(a)+1)$. Since ( $\left.1^{*}\right)$ above holds for each $a \in A$ there exist signed $f$-sequences

$$
\begin{array}{r}
\left\{\left(t(a, i, j), d^{t}\right),\left(t^{\prime}\left(a, i, j^{\prime}\right), d^{t^{\prime}}\right),\left(u(a, i, m), d^{u}\right),\right. \\
\left.\left(u^{\prime}\left(a, i, m^{\prime}\right), d^{u^{\prime}}\right): a \in A, i \in Z(n(a)+1)\right\}
\end{array}
$$

such that 
$\left(4^{\prime}\right)$

$e\left(s(a, i, r), d^{s}\right)=e\left(t(a, i, j), d^{t}\right) e\left(u(a, i, m), d^{u}\right) e\left(t^{\prime}\left(a, i, j^{\prime}\right), d^{t^{\prime}}\right)$

for $a \in A$ and $i \in Z(n(a)+1)$,

(5') $e\left(s(a, i+1, r), d^{s}\right)=e\left(t(a, i, j), d^{t}\right) e\left(u^{\prime}\left(a, i, m^{\prime}\right), d^{u^{\prime}}\right) e\left(t^{\prime}\left(a, i, j^{\prime}\right), d^{t^{\prime}}\right)$ for $a \in A$ and $i \in Z(n(a))$,

(6) $e\left(s_{a}\right)=e\left(t(a, n(a)+1, j), d^{t}\right) e\left(u^{\prime}\left(a, n(a)+1, m^{\prime}\right), d^{u^{\prime}}\right)$

$e\left(t^{\prime}\left(a, n(a)+1, j^{\prime}\right), d^{t^{\prime}}\right)$ for $a \in A$,

and such that

$$
\left(e\left(u(a, i, m), d^{u}\right), e\left(u^{\prime}\left(a, i, m^{\prime}\right), d^{u^{\prime}}\right)\right) \in R, a \in A, i \in Z(n(a)+1) .
$$

Equation $\left(2^{*}\right)$ implies there are $f$-sequences

$$
\left\{(v(a, i, p): a \in A, i \in Z(n(a)+1)\} \text { and }\left\{v\left(a, i, p^{\prime}\right): a \in A, i \in Z(n(a)+1)\right\}\right.
$$

in $S$ such that $\left(1^{\prime}\right),\left(2^{\prime}\right)$, and $\left(3^{\prime}\right)$ below hold for $a \in A$ and $i \in Z(n(a)+1)$.

$$
\begin{gathered}
v_{a, i, 1} v_{a, i, 2} \cdots v_{a, i, p(a, i)}=v_{a, i, 1}^{\prime} v_{a, i, 2} \cdots v_{a, i, p^{\prime}(a, i)}^{\prime}, \\
e\left(v_{a, i, 1}\right) e\left(v_{a, i, 2}\right) \cdots e\left(v_{a, i, p(a, i)}\right)=e\left(u(a, i, m), d^{u}\right), \\
e\left(v_{a, i, 1}^{\prime}\right) e\left(v_{a, i, 2}^{\prime}\right) \cdots e\left(v_{a, i, p^{\prime}(a, i)}^{\prime}\right)=e\left(u^{\prime}\left(a, i, m^{\prime}\right), d^{u^{\prime}}\right) .
\end{gathered}
$$

Since $g_{a} \rightarrow g$ it follows that

$$
\begin{aligned}
& k_{\delta}\left(s_{a, 1,1}\right)_{a, 1,1}^{s} k_{\delta}\left(s_{a, 1,2}\right)^{d_{a, 1,2}} \\
& k_{\delta}\left(s_{a, 1, r(a, 1)}\right)^{d_{a, 1, r(a, 1)}} \longrightarrow k_{\delta}(s) \text { for each } \delta \in D .
\end{aligned}
$$

We can now apply Property $E$ using $\left(1^{\prime}\right)-\left(7^{\prime}\right)$ to obtain that

$$
k_{\delta}\left(s_{a}\right)=k_{\hat{o}}\left(p_{R^{\prime}} e\right)^{-1} x_{a} \longrightarrow k_{\delta}(s)=k_{\delta}\left(e^{-1}(g)\right)
$$

for each $\delta \in D$. This means that $e\left(s_{a}\right) \rightarrow g=e(s)$ in $G$ and hence in $e(S)$. But $e$ is a homeomorphism and so $s_{a} \rightarrow s$ in $S$. Note that

$$
p_{R^{\prime}}\left(e\left(s_{a}\right)\right)=p_{R^{\prime}}\left(g_{a}\right)=x_{a}
$$

for each $a \in A$. Thus

$$
\left(p_{R^{\prime}} e\right)^{-1} x_{a} \longrightarrow\left(p_{R^{\prime}} e\right)^{-1} x
$$

It follows that $p_{R}, e$ is open and hence a topological isomorphism.

Now assume $S$ can be embedded in a topological group $H$ by a topological isomorphism $\psi: S \rightarrow H$. Let $H^{\prime}$ be the group generated by $\psi(S)$. As in the first part of the theorem we obtain maps $q: F(S) \rightarrow S$ and $w: F(S) \rightarrow G(S)=S$ such that $q e^{\prime}=i_{S}$ and $w e^{\prime}=e$. Again we let $R=w^{-1} \circ q \circ q^{-1} \circ w, R^{\prime}$ the congruence on $G$ generated by $R$ and $p_{R^{\prime}}: G \rightarrow G / R^{\prime}$ the natural projection. There exists a con- 
tinuous homeomorphism $\eta: G \rightarrow H^{\prime}$ such that $\eta e=\psi$ since $G$ is the free topological group generated by $S$. Now we have that $R^{\prime} \subseteq R_{\eta}=$ the congruence induced by $\eta$. (This last fact is easily shown to be true by an argument similar to the one in [1, p. 291].) There exists a continuous homeomorphism $\varphi: G / R^{\prime} \rightarrow H^{\prime}$ such that $\eta=\varphi p_{R^{\prime}}$. Hence $\psi=\eta e=\varphi p_{R^{\prime}} e$ and $p_{R^{\prime}} e$ is one-to-one since $\psi$ is one-to-one. Also, $p_{R^{\prime}} e$ is clearly continuous. Using the fact that $\psi$ is open we can show that $p_{R^{\prime}}, e$ is open by the following argument. Let $U$ be open in $S$. Then $\psi(U)$ is open in $\psi(S)$ and hence $\psi(U)=V \cap \psi(S)$ where $V$ is open in $H^{\prime}$. Now

$$
\begin{aligned}
p_{R^{\prime}} e(U) & =\varphi^{-1} \psi(U) \cap p_{R^{\prime}} e(S)=\varphi^{-1}(V \cap \psi(S)) \cap p_{R^{\prime}} e(S) \\
& =\varphi^{-1}(V) \cap \varphi^{-1} \psi(S) \cap p_{R^{\prime}} e(S)=\varphi^{-1}(V) \cap p_{R^{\prime}} e(S)
\end{aligned}
$$

which is open in $p_{R^{\prime}} e(S)$. Thus $p_{R^{\prime}} e: S \rightarrow p_{R^{\prime}} e(S)$ is open. To show that $S$ has Property $E$ assume there exist a directed set $A$, signed $f$-sequences

$$
\begin{gathered}
\left\{\left(s(a, i, r) d^{s}\right),\left(t(a, i, j), d^{t}\right),\left(t^{\prime}\left(a, i, j^{\prime}\right), d^{t^{\prime}}\right),\left(u(a, i, m), d^{u}\right),\right. \\
\left.\left(u^{\prime}\left(a, i, m^{\prime}\right), d^{u^{\prime}}\right): a \in A, i \in Z(n(a)+1)\right\},
\end{gathered}
$$

$f$-sequences

$$
\{v(a, i, p): a \in A, i \in Z(n(a)+1)\},\left\{v^{\prime}\left(a, i, p^{\prime}\right): a \in A, i \in Z(n(a)+1)\right\},
$$

a net $\left\{s_{a}, a \in A\right\}$ in $S$, and $s \in S$ such that (1)-(4), (6)-(7) in Definition 2.2 hold for each $a \in A, i \in Z(n(a)+1)$ and such that (5) in Definition 2.2 holds for each $a \in A, i \in Z(n(a))$. Condition (7) in Definition 2.2 implies that

$$
g(a)=e\left(s(a, 1, r), d^{s}\right) \longrightarrow e(s)
$$

in $G$. Hence $p_{R^{\prime}}\left(g_{a}\right) \rightarrow p_{R^{\prime}} e(s)$. Let $R_{2}$ be the congruence defined on $G$ in the first part of the proof. Equations (1), (2), and (3) in Definition 2.2 imply that

$$
\left(e\left(u(a, i, m), d^{u}\right),\left(e\left(u^{\prime}\left(a, i, m^{\prime}\right), d^{u^{\prime}}\right)\right) \in R\right.
$$

for each $a \in A$ and $i \in Z(n(a)+1)$. Equations (4), (5), and (6) in Definition 2.2 imply that

$$
\left(e\left(s(a, i, r) d^{s}\right), e\left(s(a, i+1, r), d^{s}\right)\right) \in R_{2}
$$

for each $a \in A$ and $i \in Z(n(a))$ and that

$$
\left(e\left(s(\alpha, n(\alpha)+1, r), d^{s}\right), e\left(s_{a}\right)\right) \in R_{2} .
$$

Thus

$$
\left.\left(e(s(a, 1, r)), d^{s}\right), e\left(s_{a}\right)\right)=\left(g_{a}, e\left(s_{a}\right)\right) \in R
$$


for each $a \in A$. This means that $p_{R^{\prime}}\left(g_{a}\right)=p_{R^{\prime}} e\left(s_{a}\right)$ and hence

$$
p_{R^{\prime}} e\left(s_{a}\right) \longrightarrow p_{R^{\prime}} e(s) \text {. }
$$

We have already shown that $p_{R^{\prime}} e$ is a topological isomorphism and hence $s_{a} \rightarrow s$ in $S$. Continuity of $e$ implies that $e\left(s_{a}\right) \rightarrow e(s)$ in $G$, i.e., $k_{\delta}\left(s_{a}\right) \rightarrow k_{\delta}(s)$ for each $\delta \in D$. Thus $S$ has Property $E$.

COROLlary 2.5. The completely regular topological semigroup $S$ can be embedded in a topological group if and only if $S$ can be embedded in $G / R^{\prime}$ by $p_{R^{\prime}}$ e.

CoRollary 2.6. If $N$ is a commutative, cancellative Hausdorff topological semigroup with Property $F$, then $N$ has Property $E$.

Proof. By Theorem 2.1 of [7] and its proof $N$ can be embedded in its group of quotients and $N$ is completely regular.

EXAMPLE 2.7. Let $S$ be the positive reals with the Euclidean topology. Then $S$ is embeddable as an open subset of the reals and hence has both Property $F$ and Property $E$.

ExAmple 2.8. Let $S$ be the reals with the half open interval topology. Then $S$ is embeddable algebraically in a group but not embeddable in any topological group since if $S$ were embeddable in some topological group $G$, then the image would be a subgroup of $G$ and hence $S$ would be a topological group. Thus $S$ doesn't have Property $E$ and hence doesn't have Property $F$ by 2.6 .

EXAMPLE 2.9. Let $S$ be the nonnegative reals with the Euclidean topology. Then $S$ can be embedded in $R$, the reals with the Euclidean topology and hence $S$ has Property $E$. Now $R$ is the group of quotients of $S$ and hence $S$ doesn't have Property $F$ since $S$ is not open in $R$.

EXAMPLE 2.10. If $S$ is a completely regular topological semigroup such that the embedded image of $S$ in its free topological group is open, then $S$ has Property $E$ since then $p_{R^{\prime}} e$ is open in the proof of 2.4 .

3. Subsemigroups of compact semigroups. In this section $S$ will be a commutative, cancellative, subsemigroup of a compact Hausdorff semigroup $T$ such that if $s \in S, t \in T$ and $s t \in S$, then $t \in S$. Construct the group of quotients $G$ of $S$ in the usual fashion: define a congruence relation $R$ on $S \times S$ by $((a, b),(c, d)) \in R$ if and only if $a d=b c$. Let $G=(S \times S) / R$ and $p: S \times S \rightarrow G$ be the 
natural projection. For $a$, any element of $S$, define $p: S \rightarrow G$ by $P(s)=p(s a, a)$. Rothman [7, Th. 3.1] shows that the mapping $P$ is a homeomorphism into $G$. Thus $S$ is embeddable in a topological group by a topological isomorphism if $G$ is a topological group. Rothman defines the following concept.

Definition 3.1. A semigroup $M$ has Property $J$ if $x, y \in M$, with $x \neq y$, imply there is an $a \in M$ such that $x=a y$ or $y=a x$.

Rothman shows that $G$ is a topological group if $S$ has Property $F$ or Property $J$. In the following we give a more general condition under which $G$ is a topological group.

Definition 3.2. The topological semigroup $S$ is group liftable if and only if given a convergent net $p\left(a_{\alpha}, b_{\alpha}\right) \rightarrow p(\alpha, b)$ in $G$ there exist a subnet $\left\{\left(a_{\beta}, b_{\beta}\right)\right\}$ of $\left\{\left(a_{\alpha}, b_{\alpha}\right)\right\}$ and nets $\left\{a_{\beta}^{\prime}\right\},\left\{b_{\beta}^{\prime}\right\}$ such that $\left\{\left(a_{\beta}^{\prime}, b_{\beta}^{\prime}\right)\right\}$ converges to $(x, y)$ in $S \times S$ with $x b=y a$ and such that $a_{\beta}^{\prime} b_{\beta}=b_{\beta}^{\prime} a_{\beta}$ for each $\beta$.

A continuous surjection $f: X \rightarrow Y$ of topological spaces $X$ and $Y$ will be called a decomposition map if $Y$ has the quotient topology relative to $f$, i.e., a subset $U$ of $Y$ is open if and only if $f^{-1}(U)$ is open in $X$.

THEOREM 3.3. If $S$ is group liftable, then the group of quotients $G$ of $S$ is a topological group.

Proof. Let $m, m \times m, m^{*}$ be the binary operations in $S, S \times S$, $(S \times S) / R=G$ respectively. It will be first shown that $m^{*}$ is continuous. To do this let $B$ be closed in $G$. Let the net $\left\{\left(p\left(a_{\alpha}, b_{\alpha}\right)\right.\right.$, $\left.\left.p\left(u_{\alpha}, v_{\alpha}\right)\right)\right\}$ converge to $(p(a, b), p(u, v))$ in $G \times G$ with

$$
\left(p\left(a_{\alpha}, b_{\alpha}\right), p\left(u_{\alpha}, v_{\alpha}\right)\right) \in A=m^{*-1}(B)
$$

for each $\alpha$. Since $S$ is group liftable there exist a subnet $\left\{\left(a_{\beta}, b_{\beta}\right)\right\}$ of $\left\{\left(a_{\alpha}, b_{\alpha}\right)\right\}$ and nets $\left\{a_{\beta}^{\prime}\right\},\left\{b_{\beta}^{\prime}\right\}$ such that $\left\{\left(a_{\beta}^{\prime}, b_{\beta}^{\prime}\right)\right\}$ converges to $(x, y)$ in $S \times S$ with $x b=y a$ and such that $a_{\beta}^{\prime} b_{\beta}=b_{\beta}^{\prime} a_{\beta}$ for each $\beta$. Also by the group liftability of $S$ there exist a subnet $\left\{\left(u_{\nu}, v_{\nu}\right)\right\}$ of $\left\{\left(u_{\beta}, v_{\beta},\right\}\right.$ and nets $\left\{u_{\nu}^{\prime}\right\},\left\{v_{\nu}^{\prime}\right\}$ such that $\left\{\left(u_{\nu}^{\prime}, v_{\nu}^{\prime}\right)\right\}$ converges to $(w, z)$ in $S \times S$ with $w v=z u$ and such that $u_{\nu}^{\prime} v_{\nu}=v_{\nu}^{\prime} u_{\nu}$ for each $\nu$. Then $\left(\left(a_{\nu}^{\prime}, b_{\nu}^{\prime}\right),\left(u_{\nu}^{\prime}, v_{\nu}^{\prime}\right)\right)$ converges to $((x, y),(w, z))$ in $(S \times S) \times(S \times S)$. Now $(p \times p)^{-1} A=$ $(p(m \times m))^{-1} B$ in $(S \times S) \times(S \times S)$ implies that $((x, y),(w, z)) \in(p \times p)^{-1} A$, that is, $(p(x, y), p(w, z)) \in A$. But $p(x, y)=p(a, b)$ and $p(w, z)=p(u, v)$ and so $A$ is closed in $G \times G$. Thus $m^{*}$ is continuous. 
Let $h: G \rightarrow G$ be the operation of taking inverses in $G$. Define $h^{\prime}: S \times S \rightarrow S \times S$ by $h^{\prime}\left(s, s^{\prime}\right)=\left(s^{\prime}, s\right)$ for $\left(s, s^{\prime}\right) \in S \times S$. Then we have that $p h^{\prime}=h p$. Now $p h^{\prime}$ is continuous and hence $h$ is continuous since $p$ decomposition map. Therefore $G$ is a topological group.

CoRollary 3.4. If $S$ has Property $F$ or Property $J$, then $G$ is a topological group.

Proof. Rothman [7, Lemma 1.2 and Lemma 1.4] showed that $p: S \times S \rightarrow G$ is open. Thus $S$ is group liftable.

EXAMPLE 3.5. Let $T=[0,1 / 2]$ with ordinary multiplication and the usual topology. Let $S=\{t \in T: t$ rational, $t \neq 0\}$. $S$ doesn't have Property $F$ or Property $J$, but $S$ is group liftable.

The author is indebted to the referee for helpful suggestions.

\section{REFERENCES}

1. A. H. Clifford and G. B. Preston, The algebraic theory of semigroups, Vol. II, Mathematical Surveys No. 7, Amer. Math. Soc., Providence, R.I., 1961.

2. B. Gelbaum, G. K. Kalish, and J.M. Olmstead, On the embedding of topological semigroups and integral domains, Proc. Amer. Math. Soc. 2 (1951), 807-821.

3. E. Hewitt and K. A. Ross, Abstract harmonic analysis I, Academic Press, New York, 1963.

4. S. Kakutani, Free topological groups and infinite direct product topological groups, Proc. Imp. Acad. Tokyo 20 (1944), 595-598.

5. A.I. Malcev, On the immersion of associative systems in groups, Mat. Sbornik (N.S.) 6 (1939), 331-336.

6. J. E. L. Peck, On the embedding of a topological semigroup in a topological group and its generalizations and an ergodic theorem, Dissertation, Yale Univ. 1950.

7. N. J. Rothman, Embedding of topological semigroups, Math. Ann. 139 (1960), 197-203.

8. E. Schieferdecker, Einbettungssätze für topologische Halbgruppen, Math. Ann. 131 (1956), 372-384.

Received October 24, 1969, and in revised form February 16, 1970. Some of the results in this paper were contained in the author's doctoral dissertation written at Rutgers University under Professor Louis F. McAuley.

Temple University

Philadelphia, Pennsylvania 



\title{
PACIFIC JOURNAL OF MATHEMATICS
}

\author{
EDITORS
}

\author{
H. SAMELSON \\ Stanford University \\ Stanford, California 94305 \\ RichaRd PIERCE \\ University of Washington \\ Seattle, Washington 98105
}

J. DugundJI

Department of Mathematics

University of Southern California

Los Angeles, California 9.0007

RICHARD ARENS

University of California

Los Angeles, California 9.0024

\section{ASSOCIATE EDITORS}

E. F. BeCKenBACH
B. H. NeumanN

F. WoLE

K. YoSHIDA

\section{SUPPORTING INSTITUTIONS}

\author{
UNIVERSITY OF BRITISH COLUMBIA \\ CALIFORNIA INSTITUTE OF TECHNOLOGY \\ UNIVERSITY OF CALIFORNIA \\ MONTANA STATE UNIVERSITY \\ UNIVERSITY OF NEVADA \\ NEW MEXICO STATE UNIVERSITY \\ OREGON STATE UNIVERSITY \\ UNIVERSITY OF OREGON \\ OSAKA UNIVERSITY \\ UNIVERSITY OF SOUTHERN CALIFORNIA
}

\author{
STANFORD UNIVERSITY \\ UNIVERSITY OF TOKYO \\ UNIVERSITY OF UTAH \\ WASHINGTON STATE UNIVERSITY \\ UNIVERSITY OF WASHINGTON \\ AMERICAN MATHEMATICAL SOCIETY \\ CHEVRON RESEARCH CORPORATION \\ TRW SYSTEMS \\ NAVAL WEAPONS CENTER
}

The Supporting Institutions listed above contribute to the cost of publication of this Journal, but they are not owners or publishers and have no responsibility for its content or policies.

Mathematical papers intended for publication in the Pacific Journal of Mathematics should be in typed form or offset-reproduced, (not dittoed), double spaced with large margins. Underline Greek letters in red, German in green, and script in blue. The first paragraph or two must be capable of being used separately as a synopsis of the entire paper. The editorial "we" must not be used in the synopsis, and items of the bibliography should not be cited there unless absolutely necessary, in which case they must be identified by author and Journal, rather than by item number. Manuscripts, in duplicate if possible, may be sent to any one of the four editors. Please classify according to the scheme of Math. Rev. Index to Vol. 39. All other communications to the editors should be addressed to the managing editor, Richard Arens, University of California, Los Angeles, California, 90024.

50 reprints are provided free for each article; additional copies may be obtained at cost in multiples of 50 .

The Pacific Journal of Mathematics is published monthly. Effective with Volume 16 the price per volume (3 numbers) is $\$ 8.00$; single issues, $\$ 3.00$. Special price for current issues to individual faculty members of supporting institutions and to individual members of the American Mathematical Society: $\$ 4.00$ per volume; single issues $\$ 1.50$. Back numbers are available.

Subscriptions, orders for back numbers, and changes of address should be sent to Pacific Journal of Mathematics, 103 Highland Boulevard, Berkeley, California, 94708.

PUBLISHED BY PACIFIC JOURNAL OF MATHEMATICS, A NON-PROFIT CORPORATION

Printed at Kokusai Bunken Insatsusha (International Academic Printing Co., Ltd.), 7-17, Fujimi 2-chome, Chiyoda-ku, Tokyo, Japan. 


\section{Pacific Journal of Mathematics}

\section{Vol. 34, No. $2 \quad$ June, 1970}

Shair Ahmad, On the oscillation of solutions of a class of linear fourth order

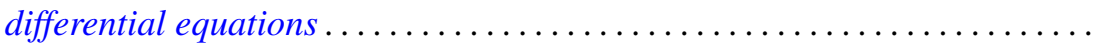

Leonard Asimow and Alan John Ellis, Facial decomposition of linearly

compact simplexes and separation of functions on cones ..............

Kirby Alan Baker and Albert Robert Stralka, Compact, distributive lattices of

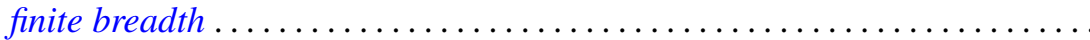

James W. Cannon, Sets which can be missed by side approximations to

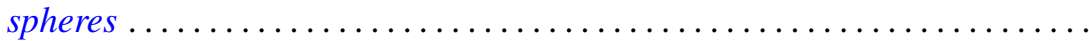

Prem Chandra, Absolute summability by Riesz means .................. 335

Francis T. Christoph, Free topological semigroups and embedding topological semigroups in topological groups....

Henry Bruce Cohen and Francis E. Sullivan, Projecting onto cycles in smooth, reflexive Banach spaces.................................

John Dauns, Power series semigroup rings .......................

Robert E. Dressler, A density which counts multiplicity ................

Kent Ralph Fuller, Primary rings and double centralizers ................

Gary Allen Gislason, On the existence question for a family of products.......

Alan Stuart Gleit, On the structure topology of simplex spaces .............

William R. Gordon and Marvin David Marcus, An analysis of equality in

certain matrix inequalities. $I \ldots \ldots \ldots \ldots \ldots \ldots \ldots \ldots$

Gerald William Johnson and David Lee Skoug, Operator-valued Feynman

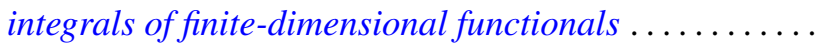

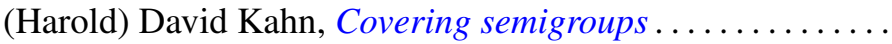

Keith Milo Kendig, Fibrations of analytic varieties

Norman Yeomans Luther, Weak denseness of nonatomic measures on perfect,

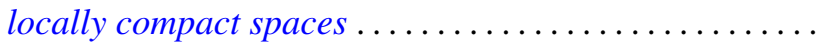

Guillermo Owen, The four-person constant-sum games; Discriminatory solutions on the main diagonal ...

Stephen Parrott, Unitary dilations for commuting contractions

Roy Martin Rakestraw, Extremal elements of the convex cone $A_{n}$ of

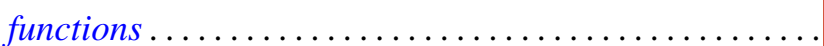

Peter Lewis Renz, Intersection representations of graphs by

William Henry Ruckle, Representation and series summability of complete

biorthogonal sequences.

F. Dennis Sentilles, The strict topology on bounded sets ...

Saharon Shelah, A note on Hanf numbers ...

Harold Simmons, The solution of a decision problem for several classes of rings. . .

Kenneth S. Williams, Finite transformation formulae involving the Legendre 